\section{Clinical Features and Predictors of Dysplasia in Proximal Sessile Serrated Lesions}

\author{
Yi Yuan Tan', Gary Sei Kiat Tay', Yu Jun Wong ${ }^{1,3}$, James Weiquan Li ${ }^{1,3}$, Andrew Boon Eu Kwek ${ }^{1,3}$, Tiing Leong Ang ${ }^{1,3}$, Lai Mun Wang ${ }^{2-4}$ \\ and Malcolm Teck Kiang Tan ${ }^{1,3}$
}

${ }^{1}$ Department of Gastroenterology and Hepatology, Changi General Hospital, Singapore, ${ }^{2}$ Department of Pathology, Changi General Hospital, Singapore, ${ }^{3}$ Yong Loo Lin School of Medicine, National University of Singapore, ${ }^{4}$ Pathology Academic Clinical Programme, SingHealth Duke-NUS Medical School, Singapore, Singapore

Background/Aims: Proximal colorectal cancers (CRCs) account for up to half of CRCs. Sessile serrated lesions (SSLs) are precursors to CRC. Proximal location and presence of dysplasia in SSLs predict higher risks of progression to cancer. The prevalence of dysplasia in proximal SSLs (pSSLs) and clinical characteristics of dysplastic pSSLs are not well studied.

Methods: Endoscopically resected colonic polyps at our center between January 2016 and December 2017 were screened for pSSLs. Data of patients with at least one pSSL were retrieved and clinicopathological features of pSSLs were analysed. pSSLs with and without dysplasia were compared for associations.

Results: Ninety pSSLs were identified, 45 of which had dysplasia giving a prevalence of $50.0 \%$. Older age (65.9 years vs. 60.1 years, $p=0.034$ ) was associated with the presence of dysplasia. Twelve pSSLs were $10 \mathrm{~mm}$ or larger. After adjusting for age, pSSLs $\geq 10 \mathrm{~mm}$ had an adjusted odds ratio of 5.98 (95\% confidence interval, 1.21-29.6) of having dysplasia compared with smaller pSSLs.

Conclusions: In our cohort of pSSLs, the prevalence of dysplasia is high at $50.0 \%$ and is associated with lesion size $\geq 10 \mathrm{~mm}$. Endoscopic resection for all proximal serrated lesions should be en-bloc to facilitate accurate histopathological examination for dysplasia as its presence warrants shorter surveillance intervals. Clin Endosc 2021;54:578-588

Key Words: Dysplasia; Large; Prevalence; Proximal; Sessile serrated lesion

\section{INTRODUCTION}

Colorectal carcinoma (CRC) is a leading cause of cancer related morbidity and mortality. There are two well established molecular pathways CRCs arise from. The adenoma-carcinoma sequence was once considered to be the only way by which CRCs develop but studies in recent years have shown that serrated lesions are also precursors to CRCs. ${ }^{1-3}$ Epidemio-

Received: July 18, 2020 Revised: December 10,2020

Accepted: December 11, 2020

Correspondence: Malcolm Teck Kiang Tan

Department of Gastroenterology and Hepatology, Changi General Hospital, 2 Simei Street 3, Singapore 529889

Tel: +65-6788-8833, Fax: +65-6788-8833, E-mail: malcolm.tan.t.k@singhealth.com.sg ORCID: https://orcid.org/0000-0001-7316-3888

(c) This is an Open Access article distributed under the terms of the Creative Commons Attribution Non-Commercial License (http://creativecommons.org/ licenses/by-nc/3.0) which permits unrestricted non-commercial use, distribution, and reproduction in any medium, provided the original work is properly cited. logical studies estimate that the serrated pathway contributes up to $30 \%$ of CRCs. ${ }^{4,5}$ Proximal CRCs account for one third to half of all CRCs and are considered biologically distinct from distal CRC. ${ }^{6-8}$ Proximal CRCs tend to occur in older persons, women and are associated with microsatellite instability (MSI) high, CpG island methylation phenotype (CIMP) positive and BRAF mutations. These are molecular features that are commonly seen in sessile serrated lesions (SSLs). ${ }^{9-11}$

Compared to adenomatous lesions, SSLs are morphologically flatter and tend towards proximal distribution (Figs. 1, 2). ${ }^{12}$ Among SSLs, proximal polyps are considered to have higher malignant potential. ${ }^{13-15}$ Serrated lesions which harbour dysplasia or are larger than $10 \mathrm{~mm}$ have been reported to carry a higher risk of progression to CRC. ${ }^{16,17}$ In a European study, IJspeert et al. regarded serrated lesions as being clinically significant if they are proximal to the splenic flexure or larger than $10 \mathrm{~mm}^{18}$

Most SSLs have somatic BRAF (V600E) mutation with 


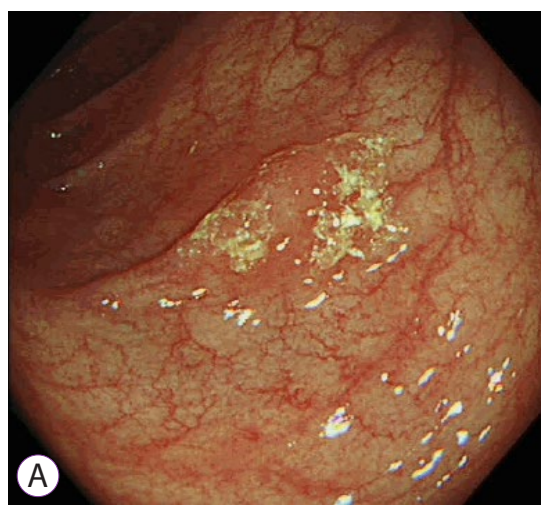

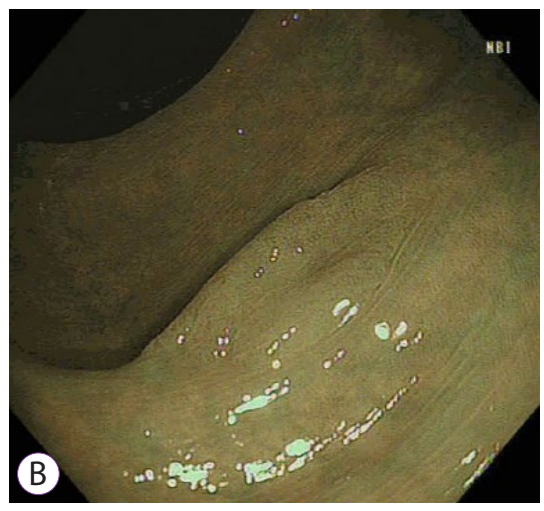

Fig. 1. Proximal sessile serrated lesion without dysplasia. White light $(\mathrm{A})$ and narrow band imaging (NBI) (B) views of a proximal sessile serrated lesion without dys Paris type 0-lla lesion covered with a mucous cap. On $\mathrm{NBI}$, the lesion has a similar colour to the back without brown vessels, findings consistent with type 1 neoplasm on narrow-band imaging international colorectal endoscopic classification.

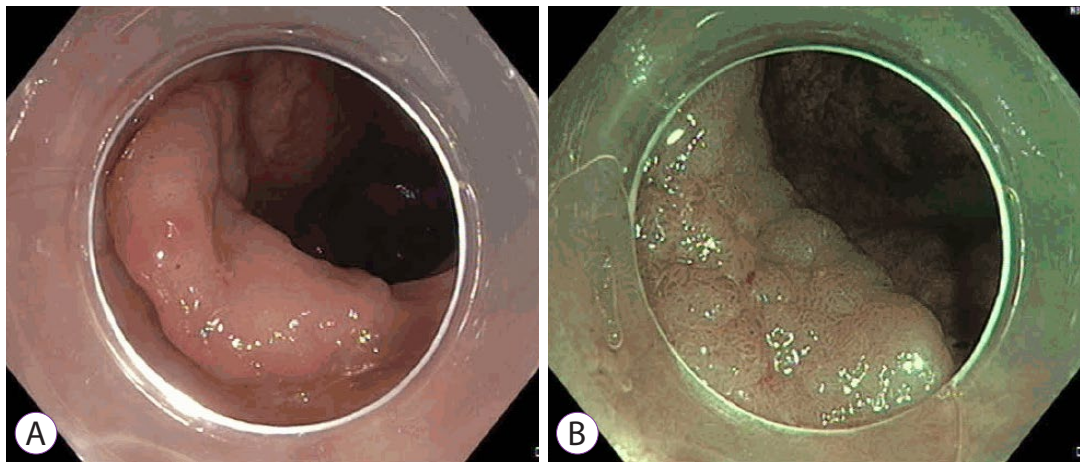

Fig. 2. Proximal sessile serrated lesion with dysplasia. White light $(\mathrm{A})$ and narrow band imaging (NBI) (B) views of a proximal sessile serrated lesion with dysplasia. Paris Type Is lesion with a nodular surface predictive of cytological dysplasia. On NBI, the lesion appears brown, with visible brown vessels and tubular branched white structures, findings consistent with type 2 neoplasm on narrow-band imaging international colorectal endoscopic (NICE) classification. Typically, NICE-2 lesions predict a histology of tubular adenoma with dysplasia.
CIMP-positive phenotype. ${ }^{19}$ The emergence of cytological dysplasia within SSLs is a critical step in carcinogenesis, as its appearance often initiates a rapid progression to malignancy. ${ }^{16,20}$ The presence of dysplasia in SSLs range from $2 \%-30 \%{ }^{21-26}$ Four patterns of dysplasia have been described, with two types having high frequencies of MLH1 expression loss, a tumour suppressor gene. ${ }^{21}$ Loss of MLH1/PMS2 is believed to initiate the transition to dysplastic change. ${ }^{27}$ This is in contrast with adenoma-carcinoma sequence, which is predominantly driven by activation in K-ras proto-oncogene, loss of p53 and APC. ${ }^{28}$ Patients with SSLs with dysplasia have higher odds of developing CRC when compared to patients with non-dysplastic SSLs. ${ }^{15}$

Due to the slow sojourn from dysplasia to malignancy, colonoscopy with polypectomy has a central role in the battle against CRC. Studies have shown that screening colonoscopy reduces the incidence for distal but not proximal CRC. ${ }^{29}$ Among patients with interval CRCs, there is a disproportionately higher contribution from lesions arising from the serrated pathway. ${ }^{30,31}$

The window of opportunity to detect and resect SSL with dysplasia is small, leading to efforts to improve colo- noscopy quality. Adenoma detection rate (ADR) has been firmly established as a quality indicator for colonoscopy performance. Recognising the significance of the serrated pathway, other measures have been proposed as a quality indicators to complement ADR, such as serrated polyp detection rate (SPDR) and proximal SPDR. ${ }^{32,33}$ While extensive literature on SSLs exists, there has been little focus on the characteristics of proximal SSLs (pSSLs). The prevalence of dysplasia in such lesions has been reported to be $8 \%-27 \%{ }^{24,34,35}$ In a retrospective study, pSSLs was associated with smoking and tended to be larger compared to distal SSLs. $^{36}$

The purpose of our study was to determine the prevalence of dysplasia in patients with pSSLs (pSSLdys) and describe the associations of these lesions.

\section{MATERIALS AND METHODS}

\section{Study design}

This was a retrospective, observational study conducted in a single tertiary referral center in Singapore. Formal Institution- 
al Review Board approval was not required as per institutional protocol for such a study design.

\section{Patient selection and data collection}

We included all patients who underwent colonoscopy between 1st January 2016 and 31st December 2017 in our hospital. A histopathology database query was performed. The histopathology slides were then reviewed by two gastrointestinal pathologists to confirm the diagnosis of SSL. A retrospective chart review of electronic medical records and electronic colonoscopy reports of patients with SSLs was then performed to collect data on patient demographics, colonoscopy and histological factors. Polyp size was determined from histology reports where possible. For polyps removed by piecemeal resection, endoscopy reports of size were recorded. In patients with more than one pSSL, we took the more clinically significant lesion (by size or presence of dysplasia) to be the index lesion.

\section{Definitions}

SSLs were diagnosed if they exhibited crypt architectural changes such as basal crypt dilation and horizontally arranged crypts along the muscularis mucosae (including 'L' or inverted ' $\mathrm{T}$ ' pattern), serrations extending to the crypt base and asymmetrical proliferation. The presence of at least 1 unequivocal architecturally distorted crypt was sufficient (Fig. 3). ${ }^{37} \mathrm{We}$ defined lesions to be proximal if they were proximal to the splenic flexure.

SSL with dysplasia was defined by the presence of abrupt transition to dysplasia reminiscent of conventional adenomas and serrated dysplasia. The dysplastic component was defined by a combination of abnormal crypt architecture (i.e., villous, crypt elongation, crowding of crypts with complex branching, cribriforming and excessive or reduced luminal serration) and cytological (intestinal type similar to conventional adenoma, serrated type characterised by round atypical nuclei, prominent nucleoli, numerous mitoses and eosinophilic cytoplasm, or subtle type such as hypermucinous change) atypia when compared to the background SSL (Fig. 4). ${ }^{37}$ Grading of dysplasia into high and low grade is not recommended for SSLs because of the heterogeneity of morphological changes precluding the achievement of satisfactory reproducibility.

Adequate bowel preparation was defined as having a Boston Bowel Preparation Score (BBPS) of at least 6 and a minimum score of 2 at each segment.

\section{Statistical analysis}

Statistical analysis was performed using SPSS version 23.0 (IBM SPSS Statistics for Windows, Version 23.0; IBM Corp., Armonk, NY, USA). Descriptive statistics were computed for all variables in frequencies and percentages. Normally distrib-

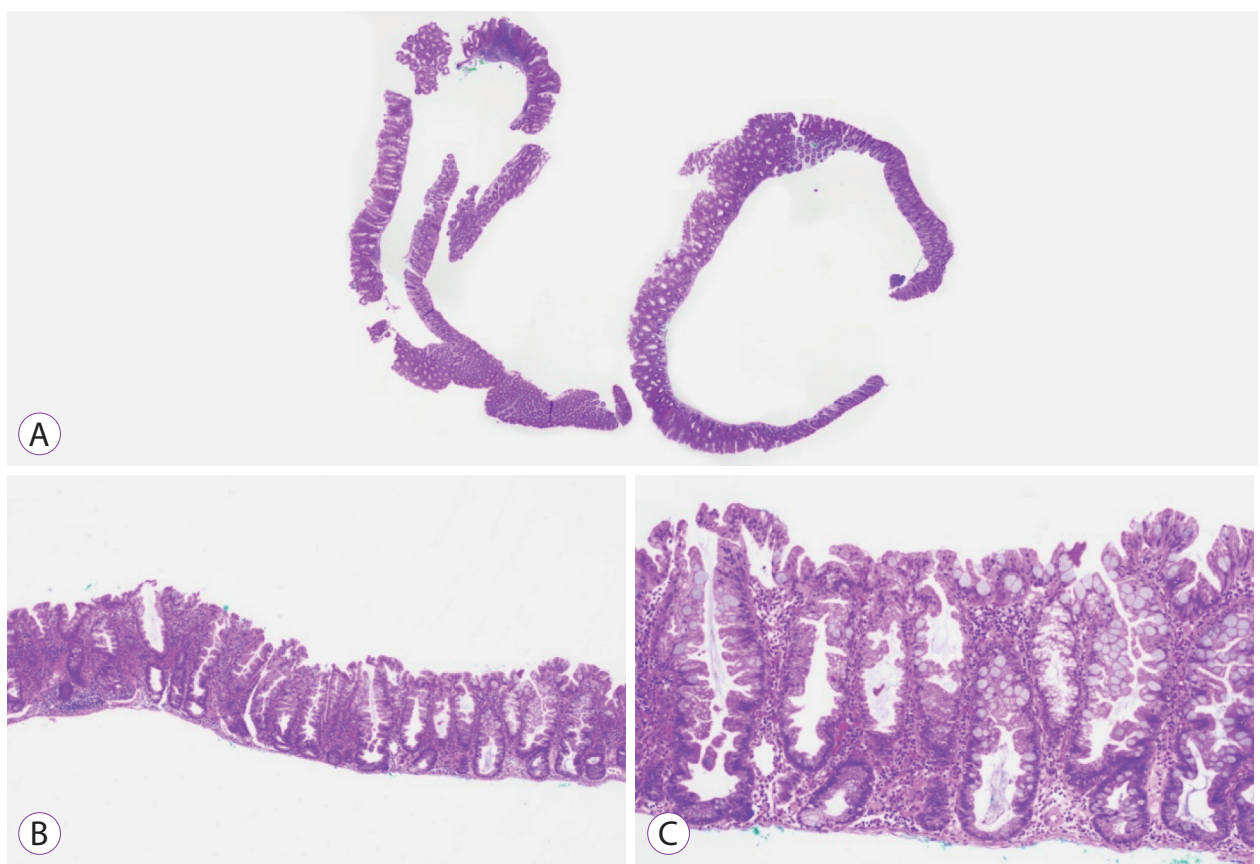

Fig. 3. Proximal sessile serrated lesion without dysplasia. (A) Hematoxylin and eosin stained section of a 5-mm proximal sessile serrated lesion without dysplasia removed by snare polypectomy. (B, C) Low and mepower microscopic views highlighting the prominent serration with extension down to the basal crypt region and the unequivocal basal crypt dilation $(\times 40, \times 100$ magnification). 


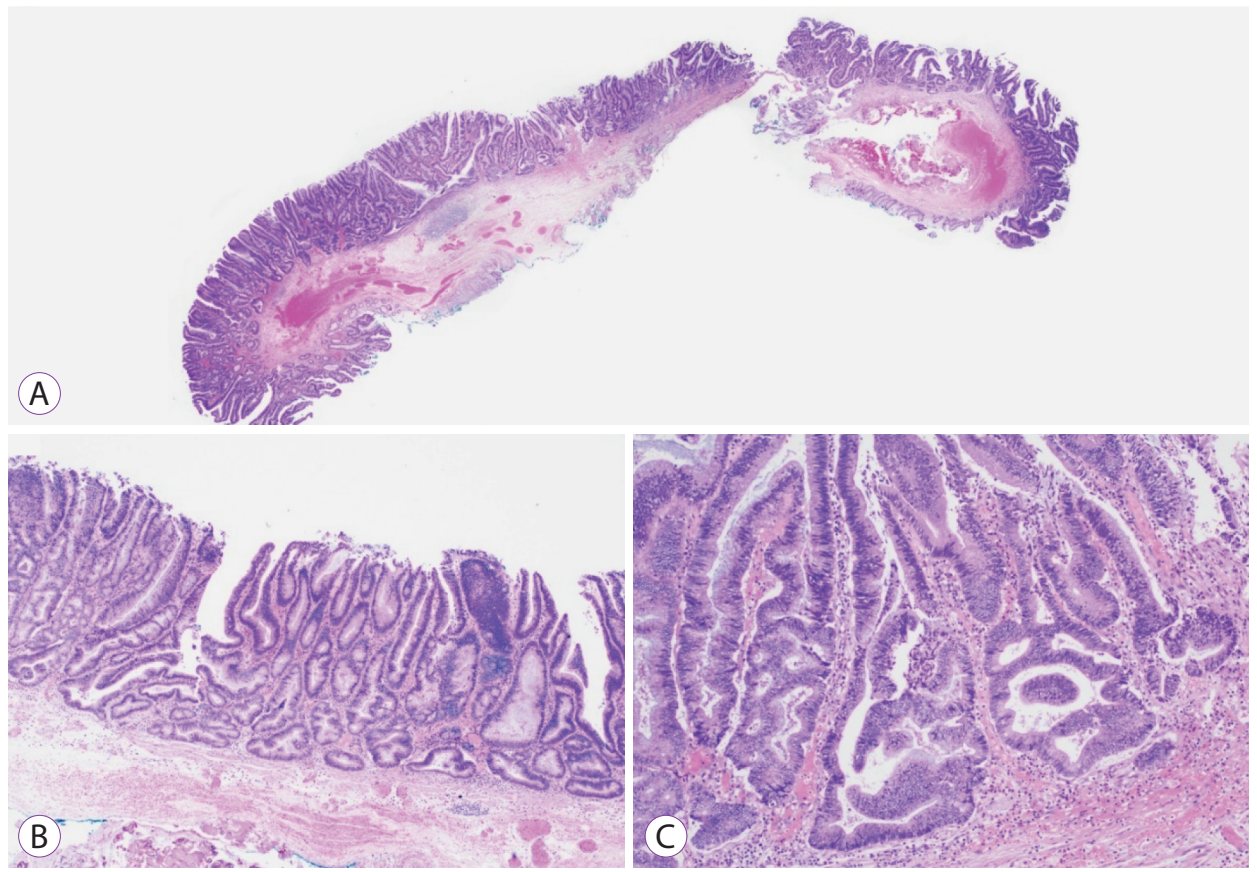

Fig. 4. Proximal sessile serrated lesion with dysplasia. (A) Hematoxylin and eosin stained section of a 20-mm proximal sessile serrated lesion with dysplasia resected en bloc by endoscopic submucosal dissection. $(B, C)$ Low and medium power microscopic views of the dysplasia resembling conventional adenoma type within the sessile serrated lesion that is defined by the charserration down to the basal crypt region with the dilation and horizontalization of the basal crypts along the muscularis mucosae $(\times 40, \times 100$ magnification).

uted continuous variables were presented in mean and standard deviation. Non-parametric variables were presented in median \pm interquartile range (IQR) and analysed using MannWhitney $U$ test. Categorical data were analysed using chisquare or Fisher's exact test. Univariate analysis was performed to identify factors associated with dysplasia. All variables with $p$-value less than 0.10 on univariate analysis were entered in multivariate analysis. Statistical significance was defined as having a $p$-value of $<0.05$.

\section{RESULTS}

\section{Baseline characteristics}

A total of 637 patients who underwent colonoscopy from January 2016 to December 2017 with histological diagnosis of proximal serrated polyps were reviewed. Our patients had an average age of $63.0( \pm 13)$ years and about half were men $(n=47,52.2 \%)$.

Out of 698 proximal serrated lesions, ninety patients had at least one lesion meeting the criteria of pSSLs, with 45 patients (50\%) having pSSLdys. Demographic features between the two groups are shown in Table 1.

We identified 90 patients with pSSLs. Compared to patients with non-dysplastic pSSLs, pSSLdys patients were older (65.9 years vs. 60.1 years, $p=0.034$ ) and were numerically more likely to have a family history of CRC $11.1 \%$ vs. $2.2 \%, p=0.101$ ) although this was not statistically significant. There was no association with other factors such as gender or indications for colonoscopy. Abdominal pain as an indication made up 17.8\% of the patients with pSSLdys, compared to $2 \%$ of patients with non-dysplastic pSSL $(p=0.015)$.

\section{Proximal sessile serrated lesion characteristics}

Median size was $4 \mathrm{~mm}$ (IQR, 3-6mm). Majority of pSSLs were found in the ascending colon (51.1\%), followed by the caecum $(26.7 \%)$, and transverse colon (22.2\%). Location of SSLs were not predictive of dysplasia $(p=0.319)$ (Table 2). Twelve polyps were $\geq 10 \mathrm{~mm}$, of which 10 (83.3\%) were dysplastic. We found that pSSLs $\geq 10 \mathrm{~mm}$ had a higher probability of having dysplasia than SSLs less than $10 \mathrm{~mm}(83 \%$ vs. $45 \%$, $p=0.013$ ). After adjusting for age, pSSLs $\geq 10 \mathrm{~mm}$ had a 6 -fold higher risk of habouring dysplasia (adjusted odds ratio $=5.98$, $95 \%$ confidence interval $=1.21-29.6)$. Even for pSSLs $<10$ $\mathrm{mm}, 35$ out of $78(44.8 \%)$ had dysplasia. Twenty seven $(60.0 \%)$ pSSLdys were smaller than $5 \mathrm{~mm}$. All of these were removed with polypectomy and most of these were found in the ascending colon $(n=17)$, followed by caecum $(n=5)$ and trans- 
Table 1. Baseline Characteristics of Patients with Proximal Sessile Serrated Lesions

\begin{tabular}{|c|c|c|c|c|}
\hline & $\begin{array}{c}\text { Total } \\
(n=90)\end{array}$ & $\begin{array}{l}\text { pSSLs with dysplasia } \\
\qquad(n=45)\end{array}$ & $\begin{array}{l}\text { pSSLs without dysplasia } \\
\qquad(n=45)\end{array}$ & $p$-value \\
\hline Age (yr), mean (SD) & $63(13)$ & $65.9(11)$ & $60.1(14)$ & 0.034 \\
\hline \multicolumn{5}{|l|}{ Gender $(n, \%)$} \\
\hline Male & $47(52.2)$ & $22(48.9)$ & $25(55.6)$ & 0.337 \\
\hline \multicolumn{5}{|l|}{ Race $(n, \%)$} \\
\hline Chinese & $66(73.3)$ & $34(75.6)$ & $32(71.1)$ & 0.560 \\
\hline Malay & $20(22.2)$ & $10(22.2)$ & $10(22.2)$ & \\
\hline Indian & $2(1.8)$ & $1(2.2)$ & $1(2.2)$ & \\
\hline Others & $2(1.8)$ & $0(0)$ & $2(4.4)$ & \\
\hline Family history of CRC $(n, \%)$ & $6(6.7)$ & $5(11.1)$ & $1(2.2)$ & 0.101 \\
\hline \multicolumn{5}{|l|}{ Smoking history } \\
\hline Yes & $20(22.2)$ & $9(20.0)$ & $11(24.4)$ & 0.305 \\
\hline No & $59(65.6)$ & $35(77.8)$ & $24(53.3)$ & \\
\hline Unknown & $11(12.2)$ & $1(2.2)$ & $10(22.2)$ & \\
\hline \multicolumn{5}{|c|}{ Indications for colonoscopy $(n, \%)$} \\
\hline Positive stool occult test & $13(14.4)$ & $8(17.8)$ & $5(11.1)$ & 0.060 \\
\hline Per rectal bleeding & $19(21.1)$ & $7(15.6)$ & $12(26.7)$ & \\
\hline Anaemia & $5(5.6)$ & $3(6.7)$ & $2(4.4)$ & \\
\hline Change in bowel habits & $24(26.7)$ & $13(28.9)$ & $11(24.4)$ & \\
\hline Abdominal pain & $9(10)$ & $8(17.8)$ & $1(2.2)$ & \\
\hline Surveillance & $15(16.7)$ & $3(6.7)$ & $12(26.7)$ & \\
\hline Weight loss & $3(3.2)$ & $2(4.4)$ & $1(2.2)$ & \\
\hline Family history of CRC & $2(2.2)$ & $1(2.2)$ & $1(2.2)$ & \\
\hline
\end{tabular}

CRC, colorectal carcinoma; pSSLs, proximal sessile serrated lesions; SD, standard deviation.

Table 2. Polyp Size and Location in Proximal Sessile Serrated Lesions with and without Dysplasia

\begin{tabular}{|c|c|c|c|c|}
\hline & $\begin{array}{c}\text { Total } \\
(n=90)\end{array}$ & $\begin{array}{c}\text { pSSLs with dysplasia } \\
\qquad(n=45)\end{array}$ & $\begin{array}{l}\text { pSSLs without dysplasia } \\
\qquad(n=45)\end{array}$ & $p$-value \\
\hline Polyp size (mm): median (IQR) & $4(3-6)$ & $4(3-8)$ & $4(3-6)$ & 0.525 \\
\hline Polyp size by category $(n, \%)$ & & & & 0.005 \\
\hline$<5 \mathrm{~mm}$ & $50(55.6)$ & $27(60.0)$ & $23(51.1)$ & \\
\hline $5-9 \mathrm{~mm}$ & $28(31.1)$ & $8(17.8)$ & $20(44.4)$ & \\
\hline$\geq 10 \mathrm{~mm}$ & $12(13.3)$ & $10(22.2)$ & $2(4.4)$ & \\
\hline Location & & & & 0.319 \\
\hline Caecum & $24(26.7)$ & $15(33.3)$ & $9(20.0)$ & \\
\hline Ascending colon & $46(51.1)$ & $20(44.4)$ & $26(4.4)$ & \\
\hline Transverse colon & $20(22.2)$ & $10(22.2)$ & $10(22.2)$ & \\
\hline
\end{tabular}

IQR, interquartile range; pSSLs, proximal sessile serrated lesions. 
verse colon $(n=5)$.

Seventy-six patients (84.4\%) had adequate bowel preparation. Mean withdrawal times were similar between pSSLdys and non-dysplastic groups. There were no associations between pSSLdys with either the timing of colonoscopy, or the involvement of trainees (Table 3). Ten patients had more than one SSL, and one patient had three SSLs. There were no patients with traditional serrated adenoma or serrated polyposis syndrome.

The presence of synchronous lesions and smoking history did not predict presence of dysplasia in proximal SSL (Tables $4,5)$. Fifteen patients (16.7\%) had synchronous advanced adenoma (AA). There was one synchronous cancer found in a patient with pSSLdys. The patient had moderately differentiated metastatic adenocarcinoma and molecular studies showed a microsatellite stable tumour with, BRAF (V600E), TP53, SMAD4, ZNRF3 mutations. These features suggest that the cancer arose from an SSL, with a traditional serrated adenoma as the intermediate lesion in carcinogenesis.

Table 3. Endoscopic Factors in Proximal Sessile Serrated Lesions with and without Dysplasia

\begin{tabular}{|c|c|c|c|c|}
\hline & $\begin{array}{l}\text { Total } \\
(n=90)\end{array}$ & $\begin{array}{l}\text { pSSLs with dysplasia } \\
\qquad(n=45)\end{array}$ & $\begin{array}{l}\text { pSSLs without dysplasia } \\
\qquad(n=45)\end{array}$ & $p$-value \\
\hline Boston bowel preparation score, median (IQR) & $6(6-9)$ & $7(6-9)$ & $6(6-9)$ & 0.923 \\
\hline Withdrawal time (minutes): median (IQR) & $15(10-26)$ & $16(11-25)$ & $15(10-28)$ & 0.536 \\
\hline Presence of trainee $(n, \%)$ & $61(67.8)$ & $31(68.9)$ & $30(66.7)$ & 0.500 \\
\hline Time of colonoscopy $(n, \%)$ & & & & 0.196 \\
\hline $\mathrm{AM}$ & $37(41.1)$ & $16(35.6)$ & $21(46.7)$ & \\
\hline PM & $53(58.9)$ & $29(64.4)$ & $24(53.3)$ & \\
\hline
\end{tabular}

IQR, interquartile range; pSSLs, proximal sessile serrated lesions.

Table 4. Synchronous Colonic Lesions in Proximal Sessile Serrated Lesions with and without Dysplasia

\begin{tabular}{lcc}
\hline & pSSLs with dysplasia & pSSLs without dysplasia \\
\hline Total & 63 & 51 \\
Hyperplastic polyps $(n, \%)$ & $15(23.8)$ & $22(43.1)$ \\
Synchronous SSLs $(n, \%)$ & $7(11.1)$ & $4(7.8)$ \\
Tubular adenoma with low grade dysplasia & $33(52.4)$ & $21(41.2)$ \\
Advance adenoma $(\geq 10$ mm, HGD or villous component) & $7(11.1)$ & $8(15.7)$ \\
Cancer & $1(1.6)$ & $0(0)$ \\
\hline
\end{tabular}

HGD, high grade dysplasia; pSSLs, proximal sessile serrated lesions; SSLs, sessile serrated lesions.

Table 5. Smoking History and Size of Proximal Sessile Serrated Lesions

\begin{tabular}{lcccc}
\hline & $\begin{array}{c}\text { Total } \\
(\boldsymbol{n}=\mathbf{7 9})\end{array}$ & $\begin{array}{c}\text { Size }<\mathbf{1 0} \mathbf{~ m m} \\
(\boldsymbol{n}=\mathbf{6 7})\end{array}$ & $\begin{array}{c}\text { Size } \geq \mathbf{1 0 ~ m m} \\
(\boldsymbol{n}=\mathbf{1 2})\end{array}$ & $\boldsymbol{p}$-value \\
\hline Smoking history & & & & 0.181 \\
Yes $(n, \%)$ & $18(22.8)$ & $17(25.4)$ & $1(8.3)$ \\
No $(n, \%)$ & $61(77.2)$ & $50(74.6)$ & $11(91.7)$ \\
\hline
\end{tabular}




\section{DISCUSSION}

This is the first study to specifically describe the unique features of proximal SSLs. Our study shows that the prevalence of pSSLdys of 50\% is higher than previously reported. ${ }^{24,34,35}$ This rate is also higher than the overall prevalence of dysplasia in all SSLs of $2 \%-30 \%{ }^{21-26}$

The preponderance of towards dysplasia in pSSLs is postulated to be multifactorial. Most of the proximal colon develops from the midgut, making it embryologically distinct from the distal colon. Compared to left sided neoplasms, right sided ones are more likely to have BRAF mutations and CIMP-positive phenotypes. ${ }^{9,38}$

Our high rate of dysplasia in pSSLs could be explained by the low detection rate of pSSLs, implying an enriched dysplasia rate with a low denominator of pSSLs. Nevertheless, this underscores the importance of diagnosing dysplasia in pSSLs. Most endoscopy societies regard SSLs with dysplasia as high risk lesions, therefore recommending surveillance colonoscopy at 3 years of index colonoscopy. ${ }^{39,40}$ The median size of pSSLdys was $4 \mathrm{~mm}$, with a mean size of 7 $\mathrm{mm}$. This contrasts with previous findings of previous reports of SSLs with dysplasia with mean sizes of 11-12 mm. ${ }^{34,41}$ These two studies included SSLs which had adenocarcinoma and high grade dysplasia, which is likely to have increased the average size of the lesions.

pSSLdys in our study tended to be larger, which is consistent with findings from other studies. ${ }^{34,35}$ In a seminal study by Burgess et al., dysplasia was found in one-third of SSLs larger than $20 \mathrm{~mm} .{ }^{42}$ Cancers are believed to develop in a stepwise fashion, from non-dysplastic lesions to dysplastic lesions and eventually to malignancy. Polyp growth within a serrated lesion can result from inactivation of tumour suppression genes (APC and RNF43) resulting activation of Wnt signalling pathway. ${ }^{43,44}$ Similar to adenomatous polyps, size predicts a higher risk subsequently developing CRC. . $^{17,45}$

Nevertheless, out of 50 diminutive pSSLs, 27 (54.0\%) had dysplasia (Figs 5 and 6). Bouwens et al. demonstrated that amongst a population of SSLs $<6 \mathrm{~mm}, 59 \%$ had dysplasia, which is similarto our findings. ${ }^{24}$ Even as large size is associated with the presence of dysplasia, our findings show that small size does not exclude dysplasia. In other words, large pSSL size as a predictor of cytological dysplasia is more specific than sensitive.
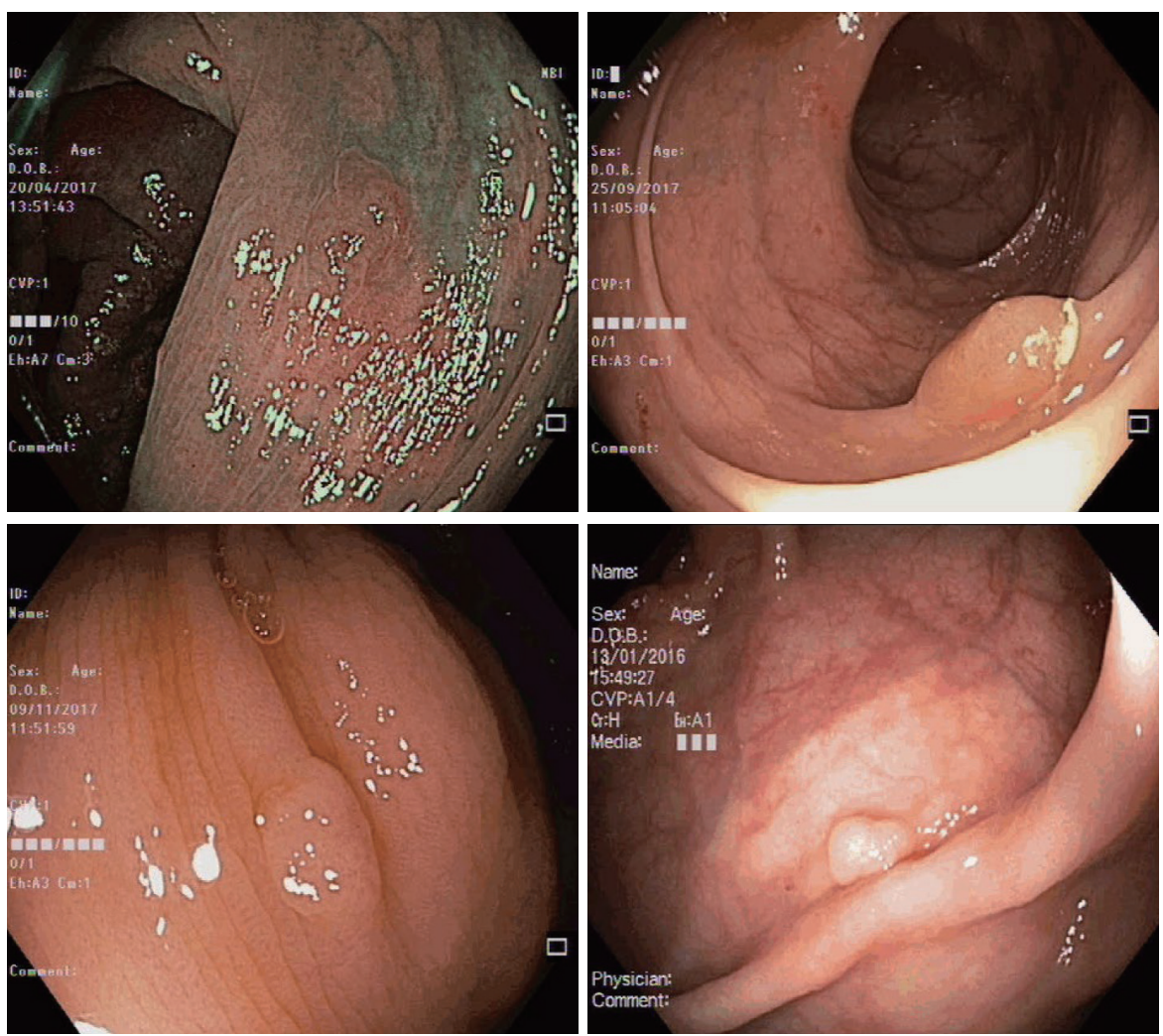

Fig. 5. Various endoscopic photos of small proximal sessile serrated lesions $(\leq 5 \mathrm{~mm})$ with dysplasia. 


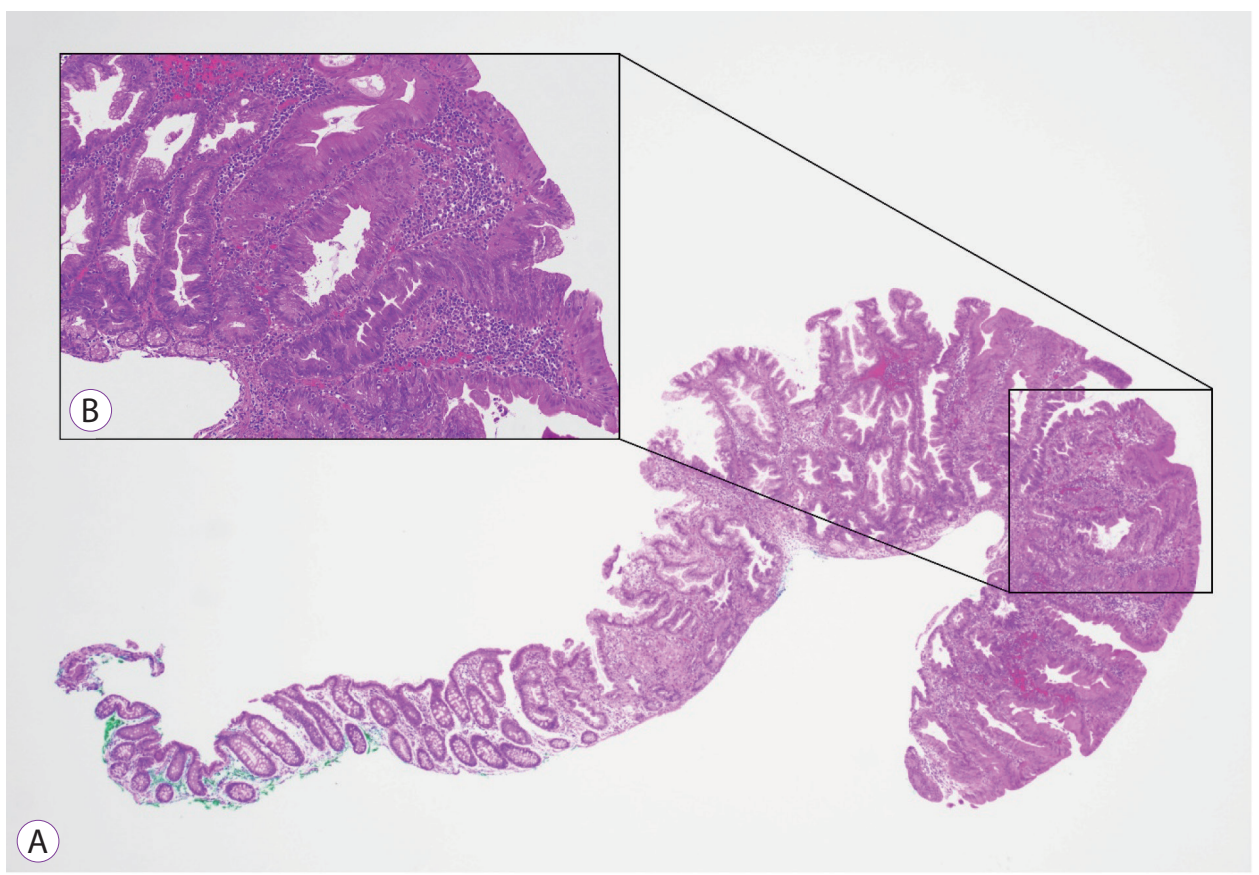

Fig. 6. Histopathology of a proximal sessile serrated lesion with dysplasia. (A) Hematoxylin and eosin stained section $(\times 20)$ of a 5 -mm proximal sessile serrated lesion with dysplasia. (B) Higher magnification of the dysplastic focus within the pSSL (×200) characterized by nuclear enlargement, hyperchromasia and pseudo stratification, changes in chromatin pattern and mitoses. pSSLs, proximal sessile serrated lesion.

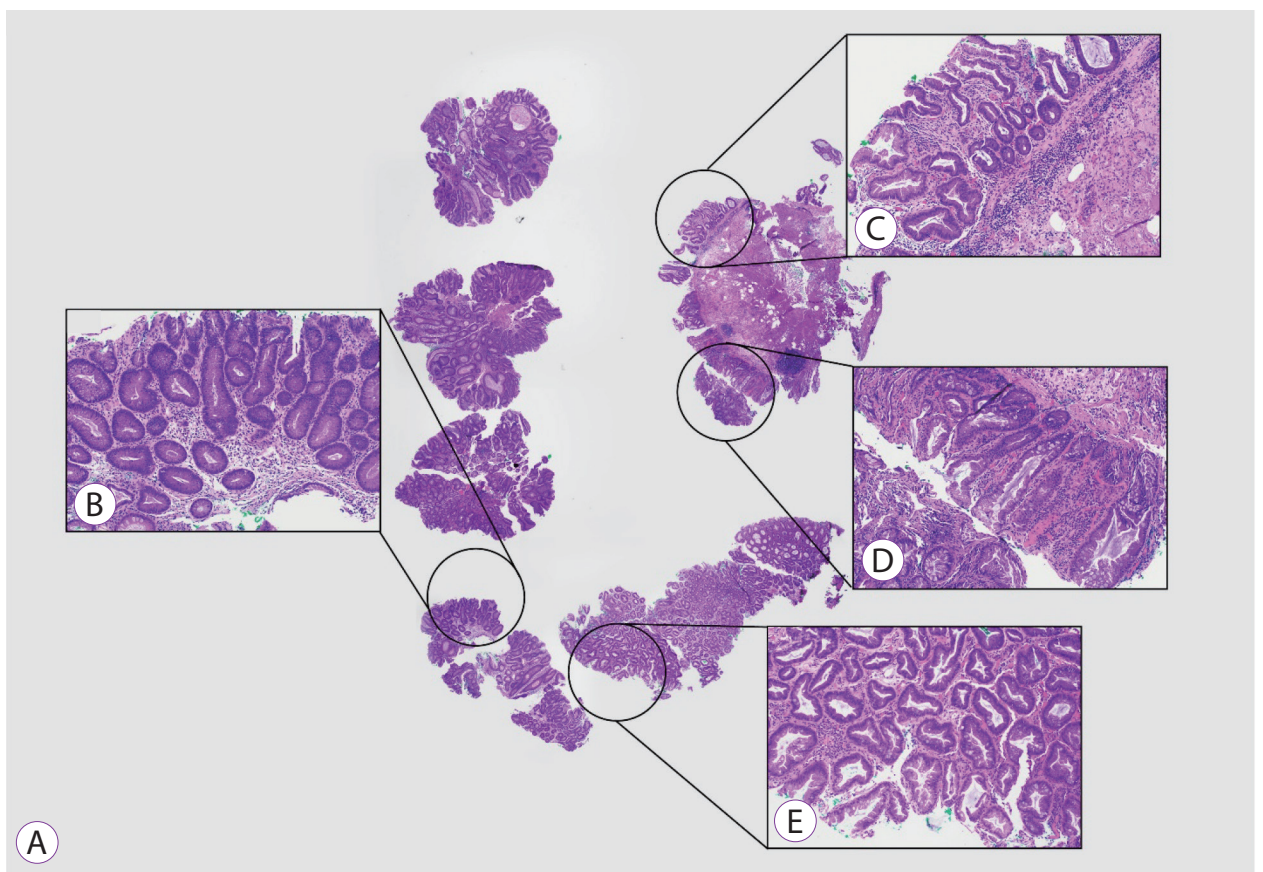

Fig. 7. Histopathology of a proximal sessile serrated lesion with dysplasia. (A) Hematoxylin and eosin stained section of a proximal sessile serrated lesion with dysplasia removed in piecemeal. (B) Medium power view of the dysplascomponent identified in a superficial fragment ( $\times 100$ magnification). (C-E) Fragments containing the characteristic histological features of sessile serrated lesion serration and basal crypt changes. Cautery artefact is present ( $\times 100$ magnification). 
A pSSLdys may be misdiagnosed as a conventional tubular adenoma when resected piecemeal (Fig. 7). The pathologist may easily misdiagnose the adenomatous component as tubulovillous or tubular adenomas and overlook the basal crypt architectural changes present in a separate fragment; and when present may also be distorted by cautery artefact precluding accurate assessment. This supports the recommendation that all lesions should be removed by en-bloc with adequate vertical margins where possible to allow for accurate histological diagnosis as crypt changes in SSLs occur at the base. ${ }^{46}$ Even for diminutive pSSLs, it is important for small lesions to be removed en-bloc with a cold snare for optimal histological assessment. Every step of the process from endoscopic assessment of serrated lesions, lesion resection, tissue handling, communication between physicians, histological assessment should be optimised for the accurate diagnosis of SSLs.

In our patients, the dysplasia occurred in patients who were on average six years older than those without dysplasia. In a group of Japanese patients with SSLs, Murakami et al. found that dysplasia in SSLs occurred in patients who were about 3 years older than those without (64.9 years vs. 62 years). ${ }^{34}$ Other published data report that although dysplasia tended to occur in older patients, there was no statistical significance. ${ }^{35,41}$ A consistent finding across all studies is that the mean age for dysplasia is around sixth and seventh decades of life.

Females in our cohort did not have a preponderance towards having pSSLdys. Out of 45 pSSLdys, 23 were found in female patients. Although there is a female predominance in pSSL, the association of dysplasia and female gender is uncertain. ${ }^{21,34,35,42}$ Sano et al. found that in 23 SSLdys, 16 were from female patients. ${ }^{35}$ In another study, 24 out 41 SSLdys were resected from female patients. Interestingly, this study also had an overrepresentation of females in SSLs with invasive carcinoma (6 out of 7), suggesting a rapid progression to cancer after onset of dysplasia. ${ }^{34}$

Location of SSLs have always been of clinical interest. Even when limited to subgroup analysis of proximal lesions, previous studies have shown a slight predilection of dysplasia to occur in SSLs found in the transverse colon. A Dutch study showed that amongst pSSLdys, 11 out of 30 (37\%) occurred in the transverse colon, compared to 16/78 (21\%) amongst non-dysplastic pSSLs. ${ }^{24}$ In a Japanese cohort, 13/34 (38\%) of pSSLdys compared to $100 / 354$ (28\%) of non-dysplasic pSSLs were located in the transverse colon. ${ }^{34}$ Our study did not show any association between anatomical location of pSSLs and the development of dysplasia. One possible explanation is small sample size with insufficient power.

Family history of CRC has not been shown to be predictive of dysplasia in SSLs. ${ }^{47}$ Although not statistically significant, there was a signal suggesting hereditability as $11.1 \%$ of our patients with pSSLdys had a family history of CRC compared with $2.2 \%$ in the non-dysplastic pSSL group $(p=0.101)$. The influence of hereditability in SSLs warrants further exploration in future studies.

Synchronous AA was found in $16.7 \%$ of our patients. The associations between AAs with both SSLs and proximal serrated lesions were previously demonstrated in average risk patients. ${ }^{48}$ One possible explanation would be that synchronous neoplasms would have similar exposure to risk factors and share molecular pathways for neoplastic change.

Our study did not show any correlation between dysplasia and smoking, which could be due to reporting or recall bias. Smoking has been shown to be associated with larger SSL size but has not been shown to be predictive of dysplasia. ${ }^{36}$ However in studies of CRC, smoking status is associated with CIMP-positive, MSI-high and BRAF-positive cancers, especially in those which are proximally located, suggesting environmental epigenetic mechanisms. ${ }^{49-51}$

Our study has some strengths. It is the first study to specifically address the issue of dysplasia in pSSLs, which has significant clinical bearing on patients as it is predictive of future risk of developing CRC. Our comprehensive histopathology database allowed us to identify all consecutive pSSLs that were diagnosed in our centre. Two gastrointestinal pathologists were involved in the double-reporting and review of histology specimens to ensure concordance in diagnosis of SSLs and dysplasia.

Nevertheless, we acknowledge that there are limitations. This is a single centre retrospective study which is prone to selection bias. The number of pSSLs resected is modest, with 90 resected from 11,822 colonoscopic procedures and this can be explained by challenges in detecting SSLs which tend to be flat, pale and covered with a mucous cap. ${ }^{30}$ Our overall detection rate of pSSLs is low, indicating the acute need for greater awareness and training of colonoscopists in detecting and resecting pSSLs. The morphological appearance of the lesions was not assessed as there were varying practices of photo-documentation by colonoscopists. As this is a retrospective study, we were unable to do perform additional histopathological studies to corroborate our clinical findings with molecular and genetic information.

In our cohort, we found that the prevalence of dysplasia in pSSLs is $50.0 \%$. After multivariate analysis, the presence of dysplasia in pSSLs is associated with lesion size $\geq 10 \mathrm{~mm}$. However, small size $<5 \mathrm{~mm}$ does not exclude dysplasia as diminutive pSSLs have a high rate of dysplasia.

Our novel findings suggest that endoscopy units should perform complete en-bloc endoscopic resection for all proximal 
serrated lesions as this would facilitate accurate histopathological diagnosis for both non-dysplastic and dysplastic SSLs. The presence of dysplasia would warrant closer surveillance intervals as it predicts higher future risk of CRC.

\section{Conflicts of Interest}

The authors have no potential conflicts of interest.

Funding

None.

\section{ORCID}

Yi Yuan Tan:

Gary Sei Kiat Tay:

Yu Jun Wong:

James Weiquan Li:

Andrew Boon Eu Kwek:

Tiing Leong Ang:

Lai Mun Wang:

Malcolm Teck Kiang Tan:

https://orcid.org/0000-0002-3572-4469 https://orcid.org/0000-0002-7965-911X https://orcid.org/0000-0002-0727-1183 https://orcid.org/0000-0002-5241-4278 https://orcid.org/0000-0003-2870-8150 https://orcid.org/0000-0001-9993-8549 https://orcid.org/0000-0001-7113-4215 https://orcid.org/0000-0001-7316-3888

\section{REFERENCES}

1. Muto T, Bussey HJ, Morson BC. The evolution of cancer of the colon and rectum. Cancer 1975;36:2251-2270.

2. Boparai KS, Dekker E, Polak MM, Musler AR, van Eeden S, van Noesel CJ. A serrated colorectal cancer pathway predominates over the classic WNT pathway in patients with hyperplastic polyposis syndrome. Am J Pathol 2011;178:2700-2707.

3. Leggett $B$, Whitehall $\mathrm{V}$. Role of the serrated pathway in colorectal cancer pathogenesis. Gastroenterology 2010;138:2088-2100.

4. Jass JR. Classification of colorectal cancer based on correlation of clinical, morphological and molecular features. Histopathology 2007;50:113130 .

5. Bettington M, Walker N, Clouston A, Brown I, Leggett B, Whitehall V. The serrated pathway to colorectal carcinoma: current concepts and challenges. Histopathology 2013;62:367-386.

6. Gomez D, Dalal Z, Raw E, Roberts C, Lyndon PJ. Anatomical distribution of colorectal cancer over a 10 year period in a district general hospital: is there a true "rightward shift"? Postgrad Med J 2004;80:667-669.

7. Baber J, Anusionwu C, Nanavaty N, Agrawal S. Anatomical distribution of colorectal cancer in a Veterans Affairs Medical Center. South Med J 2014;107:443-447.

8. Siegel RL, Miller KD, Jemal A. Cancer statistics, 2020. CA Cancer J Clin 2020;70:7-30.

9. Dekker E, Tanis PJ, Vleugels JLA, Kasi PM, Wallace MB. Colorectal cancer. Lancet 2019;394:1467-1480.

10. Missiaglia E, Jacobs B, D’Ario G, et al. Distal and proximal colon cancers differ in terms of molecular, pathological, and clinical features. Ann Oncol 2014;25:1995-2001.

11. Keum N, Giovannucci E. Global burden of colorectal cancer: emerging trends, risk factors and prevention strategies. Nat Rev Gastroenterol Hepatol 2019;16:713-732.

12. Anderson JC. Pathogenesis and management of serrated polyps: current status and future directions. Gut Liver 2014;8:582-589.

13. Kahi CJ, Hewett DG, Norton DL, Eckert GJ, Rex DK. Prevalence and variable detection of proximal colon serrated polyps during screening colonoscopy. Clin Gastroenterol Hepatol 2011;9:42-46.

14. JE IJ, de Wit K, van der Vlugt M, Bastiaansen BA, Fockens P, Dekker E.
Prevalence, distribution and risk of sessile serrated adenomas/polyps at a center with a high adenoma detection rate and experienced pathologists. Endoscopy 2016;48:740-746.

15. Erichsen R, Baron JA, Hamilton-Dutoit SJ, et al. Increased risk of colorectal cancer development among patients with serrated polyps. Gastroenterology 2016;150:895-902.e5.

16. Bettington M, Walker N, Rosty C, et al. Clinicopathological and molecular features of sessile serrated adenomas with dysplasia or carcinoma. Gut 2017;66:97-106.

17. Holme Ø, Bretthauer M, Eide TJ, et al. Long-term risk of colorectal cancer in individuals with serrated polyps. Gut 2015;64:929-936.

18. IJspeert JEG, Bevan R, Senore C, et al. Detection rate of serrated polyps and serrated polyposis syndrome in colorectal cancer screening cohorts: a European overview. Gut 2017;66:1225-1232.

19. Snover DC. Update on the serrated pathway to colorectal carcinoma. Hum Pathol 2011;42:1-10.

20. Burgess NG, Tutticci NJ, Pellise M, Bourke MJ. Sessile serrated adenomas/polyps with cytologic dysplasia: a triple threat for interval cancer. Gastrointest Endosc 2014;80:307-310.

21. Liu C, Walker NI, Leggett BA, Whitehall VL, Bettington ML, Rosty C. Sessile serrated adenomas with dysplasia: morphological patterns and correlations with MLH1 immunohistochemistry. Mod Pathol 2017;30:1728-1738.

22. Yang JF, Tang SJ, Lash RH, Wu R, Yang Q. Anatomic distribution of sessile serrated adenoma/polyp with and without cytologic dysplasia. Arch Pathol Lab Med 2015;139:388-393.

23. Abdeljawad K, Vemulapalli KC, Kahi CJ, Cummings OW, Snover DC, Rex DK. Sessile serrated polyp prevalence determined by a colonoscopist with a high lesion detection rate and an experienced pathologist. Gastrointest Endosc 2015;81:517-524.

24. Bouwens MW, van Herwaarden YJ, Winkens B, et al. Endoscopic characterization of sessile serrated adenomas/polyps with and without dysplasia. Endoscopy 2014;46:225-235.

25. Hazewinkel Y, de Wijkerslooth TR, Stoop EM, et al. Prevalence of serrated polyps and association with synchronous advanced neoplasia in screening colonoscopy. Endoscopy 2014;46:219-224.

26. Lash RH, Genta RM, Schuler CM. Sessile serrated adenomas: prevalence of dysplasia and carcinoma in 2139 patients. J Clin Pathol 2010;63:681686.

27. Sheridan TB, Fenton H, Lewin MR, et al. Sessile serrated adenomas with low- and high-grade dysplasia and early carcinomas: an immunohistochemical study of serrated lesions "caught in the act". Am J Clin Pathol 2006; 126:564-571.

28. Armaghany T, Wilson JD, Chu Q, Mills G. Genetic alterations in colorectal cancer. Gastrointest Cancer Res 2012;5:19-27.

29. Pan J, Xin L, Ma YF, Hu LH, Li ZS. Colonoscopy reduces colorectal cancer incidence and mortality in patients with non-malignant findings: a meta-analysis. Am J Gastroenterol 2016;111:355-365.

30. Nishihara R, Wu K, Lochhead P, et al. Long-term colorectal-cancer incidence and mortality after lower endoscopy. N Engl J Med 2013;369:1095-1105

31. JE IJ, Vermeulen L, Meijer GA, Dekker E. Serrated neoplasia-role in colorectal carcinogenesis and clinical implications. Nat Rev Gastroenterol Hepatol 2015;12:401-409.

32. Anderson JC, Butterly LF, Weiss JE, Robinson CM. Providing data for serrated polyp detection rate benchmarks: an analysis of the New Hampshire Colonoscopy Registry. Gastrointest Endosc 2017;85:11881194.

33. Sarvepalli S, Garber A, Rothberg MB, et al. Association of adenoma and proximal sessile serrated polyp detection rates with endoscopist characteristics. JAMA Surg 2019;154:627-635.

34. Murakami T, Sakamoto N, Ritsuno H, et al. Distinct endoscopic characteristics of sessile serrated adenoma/polyp with and without dysplasia/ carcinoma. Gastrointest Endosc 2017;85:590-600. 
35. Sano W, Fujimori T, Ichikawa K, et al. Clinical and endoscopic evaluations of sessile serrated adenoma/polyps with cytological dysplasia. J Gastroenterol Hepatol 2018;33:1454-1460.

36. Rustagi T, Rangasamy P, Myers M, et al. Sessile serrated adenomas in the proximal colon are likely to be flat, large and occur in smokers. World J Gastroenterol 2013;19:5271-5277.

37. WHO classification of tumours. Digestive System Tumours. 5th ed. Lyon: International Agency for Research on Cancer; 2019. Chapter: Colorectal serrated lesions and polyps. Pg 165-167.

38. Bufill JA. Colorectal cancer: evidence for distinct genetic categories based on proximal or distal tumor location. Ann Intern Med 1990;113:779-788.

39. Rutter MD, East J, Rees CJ, et al. British Society of Gastroenterology/Association of Coloproctology of Great Britain and Ireland/Public Health England post-polypectomy and post-colorectal cancer resection surveillance guidelines. Gut 2020;69:201-223.

40. Gupta S, Lieberman D, Anderson JC, et al. Recommendations for follow-up after colonoscopy and polypectomy: a consensus update by the US Multi-Society Task Force on Colorectal Cancer. Gastrointest Endosc 2020;91:463-485.e5.

41. Kim KH, Kim KO, Jung Y, et al. Clinical and endoscopic characteristics of sessile serrated adenomas/polyps with dysplasia/adenocarcinoma in a Korean population: a Korean Association for the Study of Intestinal Diseases (KASID) multicenter study. Sci Rep 2019;9:3946.

42. Burgess NG, Pellise M, Nanda KS, et al. Clinical and endoscopic predictors of cytological dysplasia or cancer in a prospective multicentre study of large sessile serrated adenomas/polyps. Gut 2016;65:437-446.
43. Yan HHN, Lai JCW, Ho SL, et al. RNF43 germline and somatic mutation in serrated neoplasia pathway and its association with BRAF mutation. Gut 2017;66:1645-1656.

44. Nguyen LH, Goel A, Chung DC. Pathways of colorectal carcinogenesis. Gastroenterology 2020;158:291-302

45. He X, Hang D, Wu K, et al. Long-term risk of colorectal cancer after removal of conventional adenomas and serrated polyps. Gastroenterology 2020;158:852-861.e4.

46. Kaltenbach T, Anderson JC, Burke CA, et al. Endoscopic removal of colorectal lesions-recommendations by the US Multi-Society Task Force on Colorectal Cancer. Gastroenterology 2020;158:1095-1129.

47. Davenport JR, Su T, Zhao Z, et al. Modifiable lifestyle factors associated with risk of sessile serrated polyps, conventional adenomas and hyperplastic polyps. Gut 2018;67:456-465.

48. Ng SC, Ching JY, Chan VC, et al. Association between serrated polyps and the risk of synchronous advanced colorectal neoplasia in average-risk individuals. Aliment Pharmacol Ther 2015;41:108-115.

49. Liang PS, Chen TY, Giovannucci E. Cigarette smoking and colorectal cancer incidence and mortality: systematic review and meta-analysis. Int J Cancer 2009;124:2406-2415.

50. Carr PR, Alwers E, Bienert S, et al. Lifestyle factors and risk of sporadic colorectal cancer by microsatellite instability status: a systematic review and meta-analyses. Ann Oncol 2018;29:825-834.

51. Limsui D, Vierkant RA, Tillmans LS, et al. Cigarette smoking and colorectal cancer risk by molecularly defined subtypes. J Natl Cancer Inst 2010;102:1012-1022 